\title{
Effet de l'utilisation de rations améliorées sur la rentabilité zoo-économique de l'embouche ovine paysanne en région Centre-Nord du Burkina Faso
}

\author{
Raogo Sylvain TENSABA ${ }^{1 *}$, André KIEMA ${ }^{1}$, Bila Isidore GNANDA ${ }^{1}$, \\ Aïssata WEREME N'DIAYE ${ }^{1}$, Moussa $\mathrm{ZONGO}^{2}$ et Aliou Kanfido Patoin TIEMA ${ }^{3}$ \\ IInstitut de l'Environnement et de Recherches Agricoles (INERA), 04 BP 8645 Ouagadougou 04, \\ Burkina Faso. \\ ${ }^{2}$ Université Joseph KI ZERBO, Unité de Formation et de Recherche en Science de la Vie et de la Terre \\ (UFR/SVT), Département de Biologie et Physiologie Animale (BA/PA), 03 B.P. 7021 Ouagadougou 03, \\ Burkina Faso. \\ ${ }^{3}$ Institut du Développement Rural / Université Nazi Boni, 01 BP 1091 Bobo-Dioulasso 01. \\ *Auteur correspondant ; E-mail: sylvaintensaba@yahoo.fr; Tél. : (+226) 72125080.
}

\begin{tabular}{lll}
\hline Received: 09-08-2021 & Accepted: 15-12-2021 & Published: 31-12-2021 \\
\hline
\end{tabular}

\section{RESUME}

L'embouche ovine est une activité de plus en plus pratiquée. Cependant, les résultats restent mitigés. Cette étude avait pour objectif d'améliorer les performances zoo-économiques de l'embouche ovine dans deux villages (Sian et Fanka) de la région du Centre-Nord du Burkina Faso. Trois lots de 19, 20 et 21 béliers ont été utilisés. Les animaux du lot 1 ont été alimentés aux rations paysannes. Les ovins du lot 2 ont reçu la ration améliorée composée de $50 \%$ de paille de sorgho, $20 \%$ de fanes de niébé et $30 \%$ d'Aliment Concentré embouche ovine-INERA (AC-INERA). Ceux du lot 3 ont bénéficié de la ration améliorée comprenant $50 \%$ de paille de sorgho et 50\% de AC-INERA. Les Gains Moyens Quotidiens des béliers du lot 2 (89,59 g/animal) et du lot 3 $(86,06 \mathrm{~g} / \mathrm{animal})$ ont été significativement $(\mathrm{P}<0,05)$ plus élevés que ceux du lot $1(70,65 \mathrm{~g} / \mathrm{animal})$. Une marge bénéficiaire moyenne de 10876 F CFA par tête a été dégagée avec les animaux du lot 2 contre respectivement $9474 \mathrm{~F} \mathrm{CFA}$ et $5963 \mathrm{~F} \mathrm{CFA}$ pour les ovins des lots 3 et 1. L'étude a montré qu'il est possible d'allier performance pondérale et performance économique à partir d'une meilleure valorisation des ressources locales.

(C) 2021 International Formulae Group. All rights reserved.

Mots clés : Embouche ovine, ration, performance pondérale, marge bénéficiaire.

\section{Effect of the use of improved rations on the zoo-economic profitability of sheep fattening in the North-Center region of Burkina Faso}

\begin{abstract}
Sheep fattening is an activity that is increasingly practiced. However, results remain mixed. The objective of this study was to improve the zoo-economic performance of sheep fattening in two villages (Sian and Fanka) in the North-Center region of Burkina Faso. Three groups of 19, 20 and 21 rams were used. The animals in group 1 were fed with farmer's rations. The animals in group 2 received the improved ration composed of $50 \%$ sorghum straw, 20\% cowpea tops and 30\% concentrated sheep feed from INERA. Those in group 3 benefited from the
\end{abstract}


improved ration comprising 50\% sorghum straw and 50\% concentrated sheep feed from INERA. The average daily gain of rams in group $2(89.59 \mathrm{~g} / \mathrm{animal})$ and group $3(86.06 \mathrm{~g} / \mathrm{animal})$ were significantly $(\mathrm{P}<0.05)$ higher than those in group 1 (70.65 g/animal). An average profit margin of $10876 \mathrm{~F} \mathrm{CFA}$ per head was obtained with the animals of group 2, compared to 9474 F CFA and 5963 F CFA for the sheep of groups 3 and 1, respectively. The study showed that it is possible to combine weight performance and economic performance through better use of local resources.

(C) 2021 International Formulae Group. All rights reserved.

Keywords: Sheep fattening, ration, weight performance, profit margin.

\section{INTRODUCTION}

L'élevage constitue une des bases du développement socio-économique du Burkina Faso. Il participe pour plus de $18 \%$ à la formation du Produit Intérieur Brut (MRA, 2010). L'élevage des ovins y occupe une place très importante. L'embouche ovine est une variante de l'élevage ovin. Elle valorise les animaux à diverses occasions (Tabaski, fête de fin d'année, etc.), notamment grâce à la vente dans les centres urbains (Kiema et al., 2008a). Cependant, elle est confrontée à de nombreuses contraintes qui entravent son développement dont la plus importante reste l'alimentation. En milieu rural burkinabè, les pratiques traditionnelles de rationnement alimentaire, la faible accessibilité et la qualité limitée d'un bon nombre d'intrants zootechniques horsfermes (Somda, 2001 ; Gnanda et al., 2008), ne permettent pas une expression prompte des potentialités de croissance pondérale des animaux et d'en dégager une marge bénéficiaire importante. L'alimentation constituant le principal poste des dépenses dans l'embouche (Kiema, 2008b), une bonne maîtrise des coûts de production via l'alimentation, contribuerait au renforcement des performances de la rentabilité zooéconomique de l'embouche; d'où l'importance du rationnement alimentaire. Somda (2001) et Kiema et al. (2006) ont observé que l'embouche est techniquement et économiquement rentable surtout avec des rations qui valorisent des ressources alimentaires localement disponibles dont les prix ne sont pas renchéris par la fiscalité. Cette étude avait pour but de chercher à mieux appréhender les pratiques paysannes d'embouche et à accroître les performances techniques et économiques par l'exploitation des ressources alimentaires locales et la vulgarisation de nouvelles technologies issues de la recherche.

\section{MATÉRIEL ET MÉTHODES \\ Présentation de la zone d'étude}

L'étude a été mise en œuvre dans deux villages (Sian et Fanka) de la commune de Kaya, chef-lieu de la région du Centre-Nord du Burkina Faso. Cette commune est située entre $13^{\circ} 5^{\prime}$ Latitude Nord et $1^{\circ} 05^{\prime}$ Longitude Ouest, à $100 \mathrm{~km}$ de Ouagadougou (MHU, 2013). Le climat qui y sévit est du type Nord-Soudanien avec une longue saison sèche de huit (08) mois s'étalant d'octobre à mai et une courte saison pluvieuse de quatre (04) mois, allant de juin à septembre (MHU, 2013). La moyenne pluviométrique varie entre 414,64 et 671,31 $\mathrm{mm}$ d'eau par an avec des variations notables. La végétation est composée de savanes arbustives denses et dégradées dominées par des arbustes sous lesquels est disséminé un tapis herbacé.

\section{Le matériel animal}

L'activité d'embouche a porté sur 60 béliers entiers dont 22 têtes de race Sahélienne, 23 de race Djallonké (variété mossi) et 15 têtes métissées (race Sahélienne croisée à la race Djallonké). Ces animaux appartenaient à 20 producteurs des villages de Sian et de Fanka. Ils avaient au départ de l'étude en novembre 2020 , un poids moyen de $32,09 \pm 8,47 \mathrm{~kg}$ et étaient âgés de 20 mois en moyenne. Ils ont beneficié de l'application des mesures de prophylaxie médicale qui a consisté à les vacciner contre la pasteurellose et la peste des petits ruminants et à les déparasiter (déparasitage externe et interne). Ces animaux ont été identifiés à l'aide de numéros portés sur des plaquettes en contreplaqué nouées autour du coup par une ficelle. 


\section{Les rations alimentaires}

L'alimentation des animaux a été basée sur les ressources alimentaires locales (paille et tiges de sorgho, fanes de niébé, des sons locaux, etc.) avec l'introduction d'un aliment concentré embouche ovine-INERA et de Blocs Multinutrionnels (BMN). Les BMN étaient composés d'urée (4\%), tourteau de coton (25\%), son de maïs (20\%), Piliostigma reticulatum $(12,5 \%)$, poudre d'os (11\%), sel iodé $(12,5 \%)$, kaolin $(5 \%)$ et de ciment $(5 \%)$. L'aliment concentré embouche ovine-INERA sous forme de granulés a été fabriqué à Ouagadougou, par une usine de fabrique d'aliments pour bétail sous la supervision de techniciens de l'INERA. Il est constitué de tourteau de coton (42\%), son de blé (15\%), son de maïs $(32 \%)$, drèche de bière (8\%), Prémix $(0,5 \%)$ et de coquilles d'huîtres $(2,5 \%)$. Les animaux ont été répartis en trois lots, recevant chacun un type de ration (Tableau 1).

Les animaux du lot 1 étaient alimentés selon les pratiques paysannes (rations témoins). Les quantités et les types d'aliments utilisés dans ces rations ont été du ressort de chaque emboucheur. Toutefois, des suivis réguliers effectués à des intervalles de 15 jours ont permis de dresser une typologie des aliments utilisés et d'évaluer leur contribution dans les rations endogènes appliquées ainsi que les quantités moyennes de ces rations. Les quantités d'aliments à donner aux animaux du rationnement amélioré (rations des lots 2 et 3) ont été évaluées à hauteur de $5 \%$ de leur Poids Vif (PV) et ont fait l'objet de pesée et distribution quotidienne en deux (02) séances (matin et soir). La distribution des aliments était assurée par les producteurs ou par d'autres membres de sa famille. Cela a été rendu possible par l'utilisation de mesurettes. Chaque mesurette équivaut à un poids connu de l'aliment concentré. Concernant le fourrage, des fagots/gerbes ont été constitués selon la quantité à distribuer par animal. Les quantités d'aliments à distribuer étaient ajustées tous les 15 jours. Chaque producteur a expérimenté les trois rations en affectant un animal par ration.

\section{Suivi et collecte des données}

Au cours de l'étude (60 jours de durée), toutes les unités d'embouche des producteurs concernés, ont fait l'objet de suivis périodiques tous les 15 jours, qui ont permis de collecter des données relatives aux caractéristiques des ovins (race et performances pondérales), au type de matériel et infrastructures utilisés par les producteurs et les quantités et types d'aliments distribués aux animaux ainsi que les parts des refus alimentaires par animal. Afin d'évaluer l'impact des aliments consommés sur les performances pondérales des animaux, les béliers ont été pesés une fois tous les 15 jours à l'aide d'un peson de $100 \mathrm{~kg}$ de portée et de 500 $\mathrm{g}$ de précision. Un peson à ressort de portée 50 $\mathrm{kg}$ avec une précision de $200 \mathrm{~g}$ a été utilisé pour peser les différents échantillons d'aliments sur le terrain. L'efficacité alimentaire des rations a été déterminée à travers le calcul de l'indice de consommation (IC) qui reflète la quantité d'aliment nécessaire pour réaliser un gain de poids donné. La matière sèche ingérée (MSI) a été calculée en faisant la différence entre la quantité offerte et celle refusée sur la base de la matière sèche (MS).

Des données économiques ont été collectées auprès des producteurs à l'aide d'une fiche individuelle. Il s'agit des prix d'achat des animaux et des aliments à leur charge. Tous les facteurs de production ont été évalués au prix du marché où les producteurs se sont approvisionnés. À la fin de l'opération, les produits étaient constitués des animaux (prix de vente collectés auprès des producteurs) et du fumier. Un ramassage quotidien de fumier a été réalisé auprès de chaque animal. La quantité obtenue a été pesée tous les 15 jours. A la fin de l'opération, la valeur du fumier a été estimée au prix du marché local.

\section{Analyses statistiques}

Tous les paramètres collectés ont été saisis sur Excel et analysés à l'aide du logiciel libre R. Les variables quantitatives ont été exprimées en moyenne et écart type. La comparaison des moyennes a été faite grâce au test de Student. Les différences ont été considérées statistiquement significatives au seuil de probabilité $\mathrm{p}<0,05$. En outre, les performances économiques ont été évaluées à travers la détermination des marges brutes, des marges nettes et du ratio avantage/coût par animal et par ration alimentaire. 
Tableau 1 : Composition des rations en \% des aliments distribués.

Types d'aliments

Rations (R.)

Lot 1 (témoin) Lot 2 (R. 2) Lot 3 (R. 3)

\begin{tabular}{lcc}
\hline Paille de sorgho amélioré / paille de sorgho local (\%) & 50 & 50 \\
Fanes de niébé amélioré / Fanes de niébé local (\%) & 20 & 0 \\
Aliment concentré embouche ovine INERA (\%) & 30 & 50 \\
Blocs multinutritionnels (Ad libitum) & Oui & Oui
\end{tabular}

\section{RÉSULTATS}

Typologie et contribution des aliments aux rations paysannes

En fonction des types d'aliments utilisés quotidiennement par les producteurs, il a été possible de faire des regroupements en lots correspondant à des rations. Ainsi, il a été distingué 4 types de rations paysannes (Tableau 2). La ration paysanne 1, composée de paille de sorgho ou de mil, de fanes de niébé ou d'arachide, de Sous-Produit AgroIndustriels (SPAI) et de cosses de niébé (Tableau 2), était la plus commune dans la zone d'étude $(42,1 \%$ des producteurs). Elle est suivie de la ration paysanne $2(26,31 \%)$, de la ration paysanne $3(21,05 \%)$ et enfin de la ration $4(10,52 \%)$. L'analyse de la contribution des types d'aliments dans les rations paysannes indique que les fanes de légumineuse (arachide ou niébé) et les concentrés (SPAI et sons locaux), avec des contributions respectives allant de $30,52 \%$ à $41,28 \%$ et $7,62 \%$ à $15,34 \%$, étaient communs à toutes les rations. Les pailles de sorgho ou de mil viennent en deuxième position dans le classement des constituants communs aux différentes rations mais à des proportions plus élevées $(35,66 \%$ à $46,44 \%$ ) que les fanes de légumineuses. Il y a eu aussi les cosses de niébé et des aliments moins représentatifs comme les feuilles de pastèque, la drèche de bière de mil, les foins de fourrage naturel et les gousses de Faidherbia albida. Les aliments ont été distribués deux $(26,84 \%$ des producteurs) ou trois fois $(73,15 \%)$ par jour suivant des quantités moyennes qui ont varié de 7,89 à $10,48 \mathrm{~kg}$ MS/100 kg de poids vif (Tableau 3). Il y a une différence des quantités selon les rations. Les animaux soumis aux rations paysannes 3 et 4 ont reçu les plus grandes quantités (3 à 3,05 $\mathrm{kg} / \mathrm{animal} / \mathrm{jour})$. Les plus faibles quantités ont été enregistrées avec la ration paysanne 2 (2,72 $\mathrm{kg} \pm 0,5 / \mathrm{animal} /$ jour). Cette dernière ration a été utilisée, en majorité, par des producteurs ayant reçu, au moins une fois, une formation en embouche ovine ou en technique de fauche et conservation de fourrage naturel, et dotés du matériel nécessaire (60\% des utilisateurs).

\section{Performances zootechniques}

Les résultats en termes d'évolution pondérale, ont montré un accroissement du poids des animaux des trois lots durant l'expérimentation (Tableau 4). Le Gain Moyen Quotidien (GMQ) des animaux du lot 2 (89,59 $\mathrm{g} /$ animal $)$ a été significativement $(\mathrm{P}<0,05)$ plus élevé que celui du lot 1 (70,65 g/animal). Il a été aussi enregistré une différence significative entre le GMQ des béliers du lot 3 (86,06 g/animal) et ceux du lot $1(\mathrm{P}<0,05)$. Les IC ont été de 14,$82 ; 13,56$ et 14,37 respectivement pour la ration $1 ; 2$ et 3 . Les différences n'ont pas été significatives entre les valeurs des IC des trois rations. L'analyse des performances pondérales selon la race et le type de rationnement a montré que sous l'effet des différents types de rations utilisées, les performances pondérales ont varié selon la race. Les tests de comparaison de moyennes ont montré que pour les rations 2 et 3, les GMQ des béliers de races Sahéliennes et Mossi étaient significativement différents. Il en était de même avec les races métis et mossi. Avec les rations paysannes, il n'a pas été enregistré 
une différence significative entre les différentes races. Aussi, les GMQ des béliers sahéliens des lots 2 et 3 étaient significativement différents de ceux du lot 1 . Les mêmes tendances ont été observées avec les métis des trois lots. Les ovins Djallonké (avec des GMQ de 64,97, 69,3 et 62,45 $\mathrm{g} /$ animal respectivement pour les rations 1,2 et 3) avaient moins bien exprimé leur potentialité de croissance quels que soient le type de rationnement.

En regroupant les animaux par race indépendamment des rations, il n'a pas été enregistré une différence significative en termes de GMQ entre les sahéliens (93,28 g/animal) et les ovins de race métis $(91,86$ g/animal). Cependant, les GMQ des animaux sahéliens ou métis étaient significativement plus élevés que ceux des moutons Mossi. Les valeurs des IC enregistrées n'ont pas été significativement différentes au seuil de 5\% quelle que soit la race. Elles étaient de 14,17, 13,92 et 14,50 respectivement pour les ovins sahéliens, métis et Djallonké.

\section{Analyse coût/bénéfice}

L'analyse financière réalisée sur la base d'un compte d'exploitation montre globalement que l'embouche ovine a procuré des marges bénéficiaires relativement intéressantes (Tableau 5). Pour les animaux du lot 2, une marge bénéficiaire moyenne de 10875 F CFA par tête d'ovin embouché a été dégagée avec un ratio avantage/coût de 1,2 (pour $1 \mathrm{~F}$ CFA investi dans l'embouche, le promoteur a encaissé un surplus de 0,2 F CFA). Avec les animaux du lot 3, la marge nette était de $9474 \mathrm{~F}$ CFA en moyenne et un ratio avantage/coût de 1,17. Quant aux ovins du lot 1 , il s'en est dégagé une marge bénéficiaire moyenne de $5963 \mathrm{~F}$ CFA par tête avec un ratio avantage/coût de 1,1 .

Tableau 2 : Typologie et contribution des aliments aux rations paysannes en $\%$.

\begin{tabular}{|c|c|c|c|c|}
\hline \multirow[t]{2}{*}{ Aliments } & \multicolumn{4}{|c|}{ Types de rations (contribution en \%) } \\
\hline & $\begin{array}{c}\text { Ration } \\
\text { paysanne } 1\end{array}$ & $\begin{array}{c}\text { Ration } \\
\text { paysanne } 2\end{array}$ & $\begin{array}{c}\text { Ration } \\
\text { paysanne } 3\end{array}$ & $\begin{array}{c}\text { Ration } \\
\text { paysanne } 4\end{array}$ \\
\hline \multicolumn{5}{|l|}{ I. Aliments } \\
\hline 1. Paille de sorgho ou de mil & 46,44 & & 35,66 & \\
\hline 2. Glumes de sorgho & & 15,94 & & 12,83 \\
\hline 3. Fanes (arachide ou niébé) & 30,52 & 35,33 & 30,89 & 41,28 \\
\hline 4. Foins de fourrage naturel & & 32,76 & & \\
\hline 5. Feuilles de pastèque & & & & 20,64 \\
\hline 6. Gousses (Faidherbia albida) & & & 13,81 & \\
\hline 7. Drèche de bière de mil (dolo) & & & 7,34 & \\
\hline 8. Sons locaux & & 8,32 & 12,81 & \\
\hline 9. SPAI (tourteau de coton et son de blé) & 15,34 & 7,62 & & 14,3 \\
\hline 10. Cosses de niébé & 7,67 & & & 10,91 \\
\hline 11. Tous les aliments & 100 & 100 & 100 & 100 \\
\hline Fréquence d'utilisation de la ration & 42,1 & 26,31 & 21,05 & 10,52 \\
\hline
\end{tabular}

NB : Avec la ration paysanne 2, ceux qui ont utilisé les SPAI ( $60 \%$ des producteurs) n'ont pas utilisé le son local et inversement. En ce qui concerne la ration paysanne 3, la drèche de dolo (bière de sorgho local) a été utilisé par 50\% des producteurs. 
Tableau 3 : Quantités distribuées par type d'aliment.

\begin{tabular}{lc}
\hline \multicolumn{1}{c}{ Aliments } & MS distribuée (g/animal/j) \\
\hline Paille de sorgho ou de mil & $1228,33 \pm 0,37$ \\
Glumes de sorgho & $407,14 \pm 377,95$ \\
Fanes de légumineuse (arachide ou niébé) & $950 \pm 325,32$ \\
Foins de fourrage naturel & $224 \pm 88,76$ \\
Feuilles de pastèque & $625 \pm 176,77$ \\
Gousses de Faidherbia albida & $536,66 \pm 164,41$ \\
Drèche de bière de mil (dolo) & $450 \pm 212,13$ \\
Sons locaux & $378,57 \pm 63,88$ \\
SPAI (tourteau de coton et son de blé) & $420,83 \pm 68,94$ \\
Cosses de niébé & $264,44 \pm 101,25$
\end{tabular}

MS = Matière sèche.

Tableau 4 : Performances pondérales des ovins en fonction des différents facteurs.

\begin{tabular}{|c|c|c|c|}
\hline \multicolumn{2}{|r|}{ Facteurs } & GMQ (g/j) & IC \\
\hline \multirow{3}{*}{ Effet Ration } & Lot 1 (19 ovins) & $70,65 \pm 13,03^{a}$ & $14,82 \pm 2,73^{\mathrm{a}}$ \\
\hline & Lot 2 (20 ovins) & $89,59 \pm 22,27^{b}$ & $13,56 \pm 4,62^{\mathrm{a}}$ \\
\hline & Lot 3 (21 ovins) & $86,06 \pm 25,96^{\mathrm{b}}$ & $14,37 \pm 4,35^{\mathrm{a}}$ \\
\hline \multirow{12}{*}{$\begin{array}{l}\text { Effets combinés } \\
\text { ration et race }\end{array}$} & Races sahéliennes & & \\
\hline & Lot 1 (8 ovins) & $74,52 \pm 14,08^{\mathrm{a}}$ & $15,01 \pm 2,63^{a}$ \\
\hline & Lot 2 (6 ovins) & $106,26 \pm 19,89^{b}$ & $12,78 \pm 5,18^{a}$ \\
\hline & Lot 3 (8 ovins) & $101,31 \pm 21,28^{b}$ & $14,36 \pm 4,68^{a}$ \\
\hline & Race métis & & \\
\hline & Lot 1 (4 ovins $)$ & $73,15 \pm 10,39^{a}$ & $14,62 \pm 1,38^{\mathrm{a}}$ \\
\hline & Lot 2 (7 ovins) & $98,48 \pm 16,17^{b}$ & $13,80 \pm 4,43^{\mathrm{a}}$ \\
\hline & Lot 3 (4 ovins) & $99 \pm 10,02^{b}$ & $13,43 \pm 2,36^{\mathrm{a}}$ \\
\hline & Race mossi & & \\
\hline & Lot 1 (7 ovins) & $64,97 \pm 12,18^{a}$ & $14,72 \pm 3,63^{\mathrm{a}}$ \\
\hline & Lot 2 (8 ovins) & $69,3 \pm 16,25^{\mathrm{a}}$ & $13,93 \pm 4,94^{\mathrm{a}}$ \\
\hline & Lot 3 (8 ovins) & $62,45 \pm 18,24^{\mathrm{a}}$ & $14,87 \pm 5,09^{a}$ \\
\hline \multirow{3}{*}{ Effet race } & Races sahéliennes (22 ovins) & $93,28 \pm 21,34^{a}$ & $14,17 \pm 4,13^{a}$ \\
\hline & Race métis ( 15 ovins) & $91,86 \pm 17,12^{\mathrm{a}}$ & $13,92 \pm 3,20^{\mathrm{a}}$ \\
\hline & Race mossi ( 23 ovins) & $65,54 \pm 15,46^{\mathrm{b}}$ & $14,50 \pm 4,44^{\mathrm{a}}$ \\
\hline
\end{tabular}

NB : Pour l'effet race et l'effet type de ration, les comparaisons sont par colonne. Pour les effets combinés ration et race, les comparaisons sont par colonne mais uniquement au sein de chaque race. Les moyennes portant la même lettre ne sont pas significativement différentes au seuil de $5 \%$. 
Tableau 5 : Compte d'exploitation par lot et par animal.

\section{Libellés}

Rations

\section{Coût de production}

\subsection{Charges variables}

- Prix d'achat des animaux

$\begin{array}{lll}47500 & 47142,85 & 49642,85 \\ 9190,52 & 4598,23 & 5270,87 \\ 600 & 600 & 600 \\ 57290,52 & 52341,09 & 55513,72\end{array}$

- Coût des aliments

Lot $1 \quad$ Lot $2 \quad$ Lot 3

- Coût des produits vétérinaires

$57290,52-52341,09$

55513,72

\subsection{Charges fixes}

- Amortissement matériel/équipement

$1069,52 \quad 1054,18$

1053,47

- Amortissement infrastructures/habitat

902,71

905,93

905,71

- Main d'œuvre (forfait)

1000

1000

1000

- Total charges fixes (b)

2972,23

2960,11

2959,19

- $\quad$ Charges totales $=(a+b)$

60262,76

55301,20

58472,92

\section{Produits}

- Vente animaux sur pied

$65921,05 \quad 65833,33$

67619,04

- Valeur du fumier

$$
304,79
$$

343,504392

327,78

- Total produits (c)

66225,84

66176,8377

67946,83

- $\quad$ Marge brute $=\mathbf{c}-\mathbf{a}$

8935,31843

13835,7472

12433,1

- $\quad$ Marge nette $=c-(a+b)$

5963,08265

10875,6287

9473,9

- Ratio avantage/coût $=\mathbf{c} /(\mathbf{a}+\mathbf{b})$

1,10965308

1,20952442

1,17

\section{DISCUSSION}

Les rations paysannes exploitent prioritairement les ressources alimentaires locales abondantes. Les fanes de légumineuses (arachide ou niébé) et les concentrés (SPAI et sons locaux), reconnues pour leur qualité nutritive, ont été communs à toutes les rations. L'étude s'est déroulée durant la période postrécolte, période de forte disponibilité en fanes de légumineuses, en sons locaux et en SPAI. Les quantités distribuées, 7,89 à $10,48 \mathrm{~kg}$ MS/100 kg de PV, sont comparable à celles (8 à $11 \mathrm{~kg} \mathrm{MS} / 100 \mathrm{~kg}$ de $\mathrm{PV}$ ) rapportées par Gnanda et al. (2015) sur des pratiques paysannes en embouche ovine dans la commune rurale de Korsimoro, région du Centre-Nord (Burkina Faso). Cependant, MAE (2011) a estimé qu'il est techniquement et économiquement important pour les emboucheurs de se conformer à des valeurs d'offres de 3 à $6 \mathrm{~kg}$ MS pour $100 \mathrm{~kg}$ de PV pour les ovins d'embouche en milieu tropical. Les producteurs qui ont reçu des formations en embouche ovine ou en technique de fauche et conservation de fourrage naturel parviennent à maîtriser les quantités des aliments à distribuer à leurs animaux, donc à mieux rationner.

Les résultats de l'étude ont montré une évolution globalement positive des gains pondéraux quels que soient le type de ration. Les GMQ des ovins ont été meilleurs avec les rations améliorées (rations 2 et 3 ) qu'avec la ration 1 (rations paysannes). Cela indique que les rations améliorées étaient de bonne qualité. Plusieurs auteurs dont Gnanda et al. (2005) et Gnanda et al. (2015) ont déjà rapportés les 
relatives faibles performances des rations paysannes comparativement aux rations améliorées issues de la recherche. Cela, eu égard au faible niveau de maîtrise technique des emboucheurs ruraux et la faible qualité d'un grand nombre d'intrants zootechniques hors-fermes (Somda, 2001 ; Gnanda et al., 2008). Sous la ration 1 (rations paysannes), la valeur moyenne du GMQ a été de 71,70 \pm $16,41 \mathrm{~g} /$ animal. Cette valeur est relativement supérieure à celles observées, chez des ovins embouchés à l'aide de rations paysannes, par Gnanda et al. (2015) et Kiema et al. (2008a) en milieu paysan (avec respectivement 46,65 \pm $34,53 \mathrm{~g} / \mathrm{animal}$ et $56,6 \pm 22,3 \mathrm{~g} / \mathrm{animal})$. Cette supériorité pourrait s'expliquer par la bonne disponibilité et bonne qualité des résidus de cultures utilisés, plus particulièrement ceux liés aux légumineuses telles le niébé, l'arachide, le voandzou. En effet la période novembredécembre (période à laquelle cette étude a été effectuée) correspond à celle où il y a une grande disponibilité en résidus de cultures (Zoundi et al., 2004). L'incorporation (ad libitum) des BMN dans cette étude pourrait aussi avoir eu un impact. En effet, Moujahed et al. (2003) ont indiqué que la complémentation des fourrages pauvres par les BMN améliore à la fois leur ingestion et celle de la ration entière. Montcho et al. (2016) ont également rapporté que les BMN restent un moyen pour corriger les déficiences nutritionnelles que présentent les sous-produits agricoles.

Bien qu'il n'ait pas eu de différence significative entre les GMQ des animaux du lot 2 et ceux du lot 3 , les analyses ont montré un avantage comparatif de la ration améliorée contenant les fanes de niébé (ration 2) sur la croissance pondérale des ovins. Ce constat a été déjà fait par plusieurs auteurs dont Sana et al. (2020) et Nantoumé et al. (2000). Les fanes et les cosses de niébé seraient des aliments de bonne qualités nutritives avec un bon apport à la fois énergétique et azoté (Ousseini et al., 2017). Il reste cependant que les valeurs de GMQ obtenus au cours de la présente étude, sous l'application des rationnements améliorés $(89,59 \mathrm{~g} / \mathrm{animal}$ et $86,06 \mathrm{~g} / \mathrm{animal})$, sont moins performantes que celles rapportées par Somda (2001) qui a obtenu des valeurs de GMQ de
160,67 g/animal et 132,26 g/animal avec des ovins Mossi, métis et Bali- Bali, alimentés sur une durée de 50 jours à base de rations améliorées composées de concentrés et de fourrage (fanes de légumineuses et tige de sorgho) en milieu paysan. Ils sont également en déça de celles rapportés par Kiema et al. (2008b) sur des béliers entiers dont les GMQ ont varié de 96,66 g à 162,06 g. Par contre, Gnanda et al. (2015) ont obtenu des résultats moins intéressants $(75,54 \pm 39,11 \mathrm{~g} / \mathrm{j} / \mathrm{animal})$ sur un essai, conduit dans la région du CentreNord du Burkina Faso, utilisant des rations constituées de concentrés issues de la recherche, de fourrages locaux et un complément d'appoint (BMN) dans l'embouche d'ovins Djallonké et Métis.

Une analyse focalisée sur les races montre que les ovins de race Mossi, avec un GMQ de 65,54 g, ont été moins performants comparativement aux moutons Sahéliens. En effet, les aptitudes des ovins sahéliens à mieux exprimer leurs potentialités de croissance ont été observées par plusieurs sources (Sangaré et al., 2005 ; Gnanda et al., 2015). Toutefois, d'autres auteurs dont Somda (2001) ont rapportés de meilleures performances pondérales des races métis par rapport aux sahéliennes et aux Djallonké. Les valeurs des Indices de Consommation (IC) trouvés dans cette étude sont restées élevées comparativement à celles $(11,3$ à 11,52$)$ rapportées par Zoundi et al. (2005), mais proches de celles $(15,31$ et 14,11$)$ observées par Kima (2008) et meilleures aux IC (18,9 et 24) obtenus par Gnanda et al. (2015) avec des métis et des moutons Mossi.

L'analyse des performances pondérales selon les effets combinés race et type de ration alimentaire suggère que les performances des animaux ne sont pas influencées par un seul facteur. Ce constat a déjà été fait par Somda (2001) qui a rapporté que les performances pondérales des animaux sont plutôt tributaires de l'effet combiné de plusieurs paramètres dont la ration alimentaire et la race des animaux.

L'analyse financière réalisée sur la base d'un compte d'exploitation a montré que toutes les rations testées ont été économiquement rentables, avec une longueur d'avance de la 
ration 2. Ces résultats sont supérieurs à ceux rapportés par Toléba et al. (2001) et par Boly et al. (2001) qui ont respectivement, enregistré des marges bénéficiaires de 2325 et de - 9850 F CFA. Les charges liées à l'alimentation ont influencé les marges bénéficiaires. Les lots ayant les coûts d'alimentation les plus élevées ont dégagé des marges nettes relativement réduites.

\section{Conclusion}

Les résultats en termes de GMQ et de IC ont confirmé que les performances pondérales sont influencées par la conjugaison de plusieurs facteurs dont la race et le type de ration. Il faut en tenir compte pour améliorer la rentabilité économique. L'utilisation des rations améliorées a aussi montré qu'il est possible d'allier performance pondérale et performance économique. Une bonne maîtrise des charges liées à l'alimentation par une meilleure valorisation des ressources alimentaires locales contribuerait à améliorer les performances économiques de l'embouche. La vulgarisation de ces rations contribuera à la valorisation des ressources locales dans la production de la viande ovine.

\section{CONFLIT D'INTERETS}

Les auteurs déclarent n'avoir aucun conflit d'intérêts.

\section{CONTRIBUTIONS DES AUTEURS}

RST a été chargé de la collecte, du traitement des données et de l'écriture de manuscrit. AKPT a participé à la collecte des données. AK, BIG, MZ et AWN ont assuré le suivi de l'étude depuis la conception jusqu'à sa réalisation afin de lui garantir une qualité scientifique.

\section{REMERCIEMENTS}

Les auteurs adressent leurs vifs remerciements aux producteurs qui ont participé à la conduite des activités.

\section{REFERENCES}

Ayantunde AA, Fernandez-Rivera S, DanGomma A. 2008. Sheep Fattening with Groundnut Haulms and Millet Bran in the
West African Sahel. Revue Élev. Méd. Vét. Pays Trop., 61 (3-4): 215-220.

Boly H, Ilboudo JB, Ouédraogo M, Berti F, Lebailly P, Leroy P. 2001. L'élevage $\mathrm{du}$ "mouton de case" : aspects techniques, socio-économiques et perspectives d'amélioration au Yatenga (Burkina Faso). Biotechnol. Agron. Soc. Environ., 5 (4) : 201-208.

Gnanda B, Nianogo AJ, Zoundi JS, Somda J, Koanda S. 2005. Performances techniques et économiques de l'embouche ovine en exploitation traditionnelle de la région sahélienne au Burkina Faso. Rev. CAMES, 03 : 49-56

Gnanda $B$, Nignan $M$, Ouédraogo $S$, Werème N'diaye A, Traoré O, Sinon B. 2015. Influence d'une co-construction de rationnement amélioré sur les performances d'embouche ovine paysanne dans la commune rurale de Korsimoro au Burkina Faso. Int. J. Biol. Chem. Sci., 9(3): 1544-1556. DOI: http://dx.doi.org/10.4314/ijbcs.v9i3.35

Kima, AS. 2008. Valorisation des gousses de Piliostigma thonmngii (Schum.) en production animale et étude de l'infestation par des insectes. Mémoire d'ingénieur du développement rural, option élevage, Institut du Développement Rural (IDR), Université polytechnique de Bobo-Dioulasso, Burkina Faso, 96 p.

Kiema A, Nianogo A J, Somda J, Ouédraogo T. 2006. Valorisation des fanes de niébé et des gousses d'Acacia raddiana dans l'embouche ovine en Région Sahélienne du Burkina Faso. Etudes et Recherches Sahéliennes, 12 : 58-64.

Kiema A, Nianogo A J, Somda J, Ouédraogo T. 2008a. Valorisation des ressources alimentaires locales dans l'embouche ovine paysanne. Performances technicoéconomiques et options de diffusion. Cahiers Agricultures, 17 : 24-27. DOI: 10.1684/agr.2008.0154

Kiema A, Nianogo A J, Somda J, Ouédraogo T. 2008b. Valorisation de Cassia obtusifolia L. dans l'alimentation des ovins d'embouche en Région sahélienne 
du Burkina Faso. Tropicultura, 26 (2) : 98-103.

Kondombo S R, Nianogo A J. 2001. Performance d'ovins Djallonké alimentés à base de résidus de récolte au Burkina Faso. Agronomie Africaine, 13 (2) : 5966.

Ministère de l'Agriculture et de l'Elevage (MAE). 2011. Référentiel technicoéconomique de l'embouche ovine commerciale. $37 \mathrm{p}$.

MHU (Ministère de l'Habitat et de l'Urbanisme), 2013. Schéma Directeur d'Aménagement et d'Urbanisme de la ville de Kaya. Rapport d'étude, 227 p.

Montcho M, Babatounde S, Aboh AB, Bahini MJD, Chrysostome CA, Mensah GA. 2016. Caractéristiques physiques et nutritionnelles des blocs multi nutritionnels fabriqués à partir des sousproduits agricoles et agroindustriels du Bénin. Int. J. Biol. Chem. Sci., 10(6): 2485-2496.

DOI: http://dx.doi.org/10.4314/ijbcs.v10i6.7

Moujahed N, Kayouli C, Raach-Moujahed A. 2003. La complémentation des fourrages pauvres par les blocs multinutritionnels chez les ruminants. Principes de base et aspects pratiques. Livestock Research for Rural Development, 15(36). http://www.lrrd.org/lrrd15/5/mouj155.ht m.

MRA (Ministère des Ressources Animales). 2010. Politique Nationale de Développement Durable de l'Elevage au Burkina Faso 2010-2025, 54 p.

Nantoume H, Kouriba A, Togola D, Ouologuem B. 2000. Mesure de la valeur alimentaire de fourrages et de sousproduits utilisés dans l'alimentation des petits ruminants. Revue Elev .Méd.Vét. Pays Trop, 53 (3): 279-284.
Ousseini M, Chaibou M, Mani M. 2017. Pratique et utilisation des sous-produits de légumineuse dans l'alimentation du bétail à la communauté urbaine de Niamey : Cas de fanes et cosses de niébé (Vigna Unguiculata). Journal of Applied Biosciences, 120: 12006-12017. DOI: https://dx.doi.org/10.4314/jab.v120i1.3

Sana Y, Sangaré M, Tingueri BL, Sanou J, Sawadogo L, Kaboré-Zoungrana CY. 2020. Effet de l'utilisation de quatre rations à base de Panicum maximum sur les performances zootechniques des ovins de race Djallonké, à l'Ouest du Burkina Faso. Rev. Ramres, 08 (02): 38-44.

Somda J. 2001. Performances zootechniques et rentabilité financière des ovins en embouche au Burkina Faso. Biotechnol. Agron. Soc. Environ, 5 (2): 73-78.

Tadesse M. 2007. The influence of age and feeding regimen on the carcass traits of Arsi-Bale goats. Livestock Research for Rural Development, 19(47). DOI: http://www.lrrd.org/lrrd19/4/tade19047.h tm.

Toleba SS, Babatounde S, Trougnin H, Chabi LW, Adandedjan CC. 2001. Etude comparative de deux espèces fourragères (Panicum maximum local et Brachiaria riziziensis) complémentées par des graines de coton sur les performances pondérales des ovins Djallonké. Annales des Sciences Agronomiques du Bénin, 2: 193-208.

Zoundi JS, Nianogo AJ, Sawadogo L. 2002. Utilisation optimale des ressources alimentaires localement disponibles pour l'engraissement des ovins au sein des exploitations mixtes agriculture-élevage du Plateau Central du Burkina Faso. Revue Élev. Méd. Vét. Pays Trop., 55 (1): 53-62. 